\title{
Multifractal based image processing for estimating the complexity of COVID-19 dynamics
}

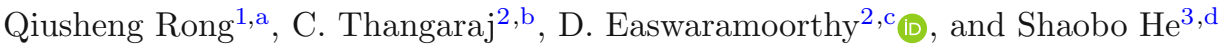 \\ 1 School of Computer and Electrical Engineering, Hunan University of Science and Arts, Changde 415000, China \\ ${ }^{2}$ Department of Mathematics, School of Advanced Sciences, Vellore Institute of Technology, Vellore, Tamil Nadu, India \\ 3 School of Physics and Electronics, Central South University, Changsha 410083, China
}

Received 8 September 2021 / Accepted 30 October 2021 / Published online 18 November 2021

(C) The Author(s), under exclusive licence to EDP Sciences, Springer-Verlag GmbH Germany, part of Springer Nature 2021

\begin{abstract}
The COVID-19 pandemic creates a worldwide threat to human health, medical practitioners, social structures, and finance sectors. The coronavirus epidemic has a significant impact on people's health, survival, employment, and financial crises; while also having noticeable harmful effects on our environment in a short span of time. In this context, the complexity of the Corona Virus transmission is estimated and analyzed by the measure of non-linearity called the Generalized Fractal Dimensions (GFD) on the chest $\mathrm{X}$-Ray images. Grayscale image is considered as the most important suitable tool in the medical image processing. Particularly, COVID-19 affects the human lungs vigorously within a few days. It is a very challenging task to differentiate the COVID-19 infections from the various respiratory diseases represented in this study. The multifractal dimension measure is calculated for the original, noisy and denoised images to estimate the robustness of COVID-19 and other noticeable diseases. Also the comparison of COVID-19 $\mathrm{X}$-Ray images is performed graphically with the images of healthy and other diseases to state the level of complexity of diseases in terms of GFD curves. In addition, the Mean Absolute Error (MAE) and the Peak Signal-to-Noise Ratio (PSNR) are used to evaluate the performance of the denoising process involved in the proposed comparative analysis of the representative grayscale images.
\end{abstract}

\section{Introduction}

A fractal is a rough or fragmented geometric shape of natural features as well as other complex objects in which the traditional Euclidean geometry fails to analysis. According to Mandelbrot, the Euclidean geometry was not enough to determine the irregularity of the objects like mountains, clouds and other complex sets. Therefore, the complex related objects can be delineated with respect to the non-linear settings called the fractal geometry. The fractal geometry is an advanced approach to characterize the complex nature by using the property of self-similarity, which was originally explored by Mandelbrot in 1975. The fractal was derived from the Latin word 'fractus', which means broken or fractured. Mandelbrot defined a fractal mathematically as a set with Hausdorff dimension strictly exceeds its topological dimension [1-6].

The nonlinear measure, fractal dimension estimates the irregularity of the given object with the various scaling properties. The concept of fractal dimension can be applicable in the measurement and categorization

\footnotetext{
${ }^{\mathrm{a}}$ e-mail: rongqs@huas.edu.cn

b e-mail: ctrmath1@gmail.com

c e-mail: easandk@gmail.com (corresponding author)

d e-mail: heshaobo_123@163.com
}

of shape and texture. Numerous research works have been described in medical image analysis employed with fractal theory. The fractal dimension is insufficient to characterize the object having complex and inhomogeneous scaling properties. The monofractal and correlation dimensional measurements are the most accessible non-linear technique in dealing with the experimental images. The single-dimensional quantity is insufficient to characterize the non-uniformity or inhomogeneity of the image. This dimensional size is not sufficient to classify the randomness or inconsistency of the experimental image. Generally, the chaotic attractors are inhomogeneous. Such an inhomogeneous set is called a Multifractal and is characterized by the Generalized Fractal Dimensions (GFD) or Renyi Fractal Dimensions. Therefore, the generalized fractal dimension is used to analyze, characterize and estimate the dynamics of complex signals and images [7-25].

In general, the photographic electronics and the light waves create the image. The existence of noise in grayscale image is regarded a defect and the following image processing techniques, such as feature extraction, image classification, and pattern recognition; are performed by using the grayscale images. The improvement of image quality is one of the most important process in image analysis. In image processing, the difficult task is 
Fig. 1 Sample X-Ray images from a healthy person and COVID-19 patient

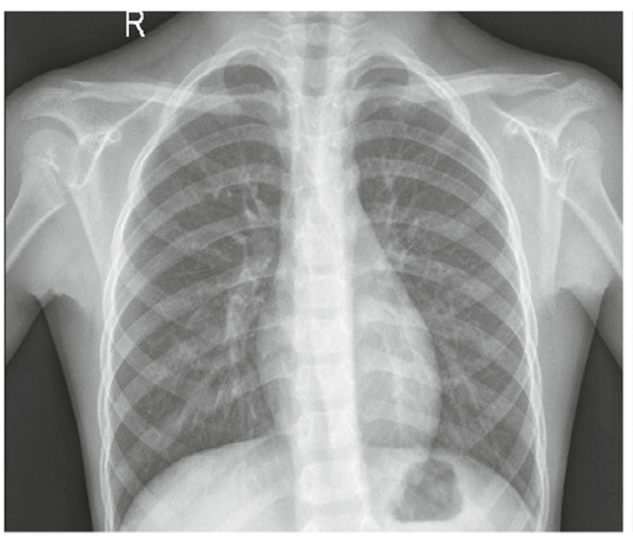

(a) Normal X-Ray Image

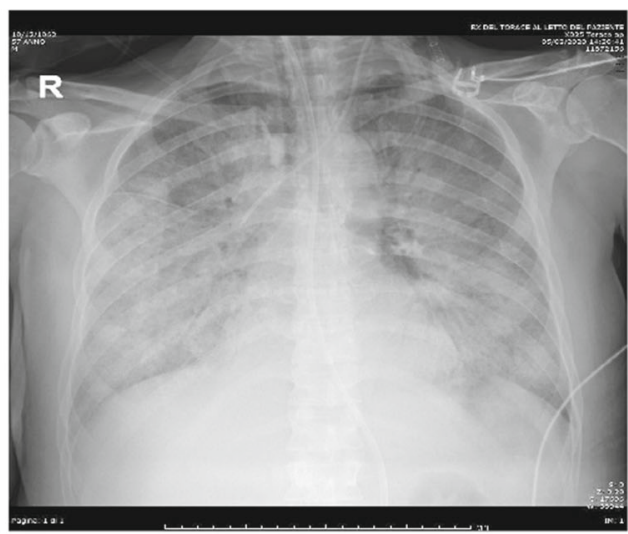

(b) COVID-19 X-Ray Image

$$
S_{q}=\frac{1}{1-q} \log _{2}\left(\sum_{i=1}^{N} p_{i}^{q}\right)
$$

using the less memory capacity [26-28].

Nowadays, COVID-19 is a lethal infectious virus and threatening the individual's life. It is rapidly spreading across the globe. This sickness has symptoms that are similar to those of the flu, such as dry cough, fever, and, its severe impact can cause difficulty in breathing [29]. Also the COVID-19 affects the human lungs directly within a week and leads to death [30]. X-Ray images are very helpful to detect the level of infections in human lungs. Moreover, X-Ray grayscale images are complex in their structures, it is not easy to differentiate the COVID-19 from the other respiratory diseases, as the symptoms are almost similar. [31-37]. In this case, the multifractal measures are used in representative images to classify the level of complexity of the diseases considered in this research study. It is assured that the roll of the GFD will be very efficient in the image analysis in order to detect the noise level in the complex images.

This paper is organized with five sections including the Introduction as in this Sect. 1. Section 2 contains the definitions and methods related to Renyi Entropy, Generalized Fractal Dimensions, Median Filter and Qualitative Measures. Data used in this study is described in Sect. 3. Experimental results are discussed in Sect. 4. Section 5 reveals the concluding statements.

\section{Methods}

The basic concepts and methods required for the research study, are discussed elaborately in this section.

\subsection{Renyi entropy}

Renyi Entropy was crucial in the development of probability theory. Renyi Entropy, a mainstreaming of Shannon Entropy, is one of a family of functionals used to assess a system's diversity, uncertainty, or unpredictability. The generalized entropy of a given probability distribution is also known as Renyi Entropy [7,8,12].

The Renyi Entropy of order $q(\neq 1)$, where $q \in \mathbb{R}$, of the given probability distribution is characterized as where $p_{i} \in[0,1]$ are the probability of $x_{i}, i \in$ $\{1,2, \ldots, N\}$.

\subsubsection{Some particular cases}

(a) If $q=0$, then $S_{0}=\log _{2} N$, the Hartley Entropy of the probability distribution.

(b) If $q \rightarrow 1$, then $S_{q}$ converges to $S_{1}$, which is defined as $S_{1}=-\sum_{i=1}^{N} p_{i} \log _{2} q_{i}$. This is the wellknown entropy of a discrete probability distribution known as Shannon Entropy.

(c) Commonly renyi entropy refers to the case $q=2$, $S_{2}=-\log _{2}\left(\sum_{i=1}^{N} p_{i}^{2}\right)$.

(d) As $q \rightarrow-\infty$, then $S_{-\infty}=-\log _{2}\left(\max \left\{p_{1}, p_{2}, \ldots\right.\right.$, $\left.\left.p_{N}\right\}\right)$ is called the maximum-entropy.

(e) As $q \rightarrow \infty$, then $S_{\infty}=-\log _{2}\left(\min \left\{p_{1}, p_{2}, \ldots, p_{N}\right\}\right)$ is called the minimum-entropy.

\subsection{Generalized fractal dimensions for gray scale images}

In this section, the multifractal analysis is described to determine the noise level of grayscale images by using Generalized Fractal Dimensions (GFD) [12-16].

The following construction is used to introduce a probability distribution of a tested grayscale image.

Let $N$ be the number of boxes and $\varepsilon$ be the box size of the given image, and the probability $p_{i}$ for the $i$ th box with $\varepsilon$ size in the tested image, is constructed as

$$
p_{i}=\frac{M_{i}}{M}
$$

where $M_{i}$ is the mass of the tested grayscale image included in the corresponding $i$ th box of size $\varepsilon$ and $M$ is the total mass of the tested grayscale image. 
Fig. 2 Sample X-Ray images from pneumonia patients

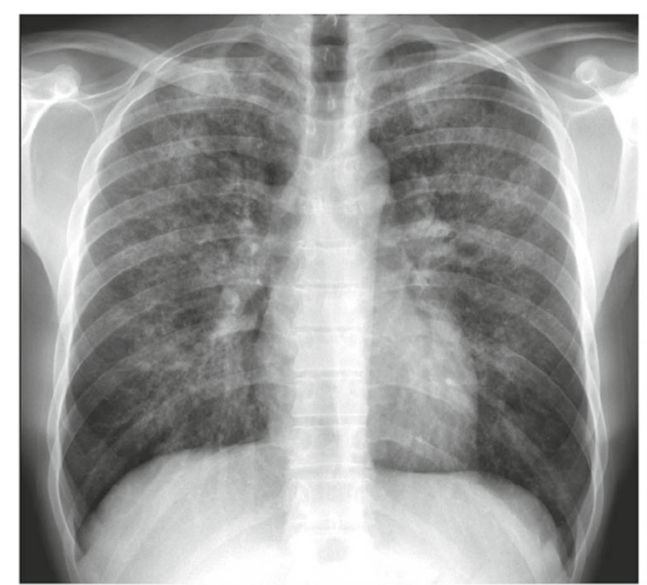

(a) Pneumocystis Pneumonia

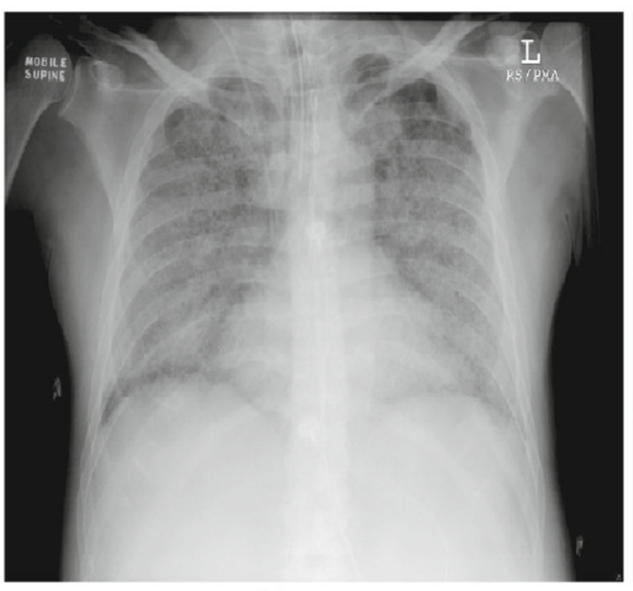

(c) ARDS

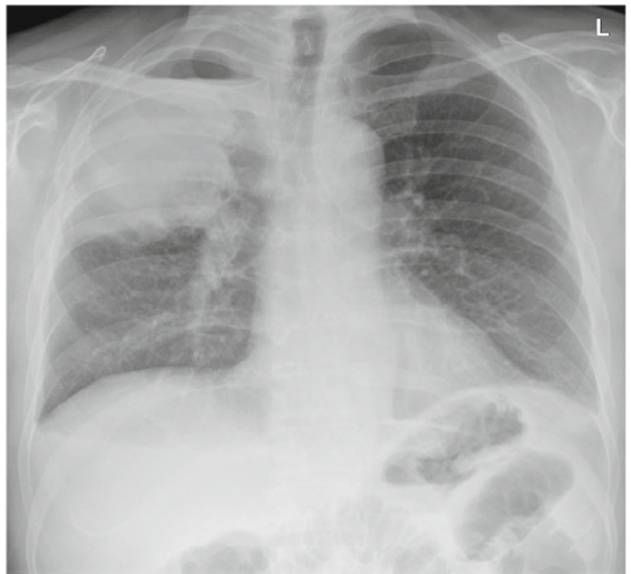

(e) Cavitating Pneumonia

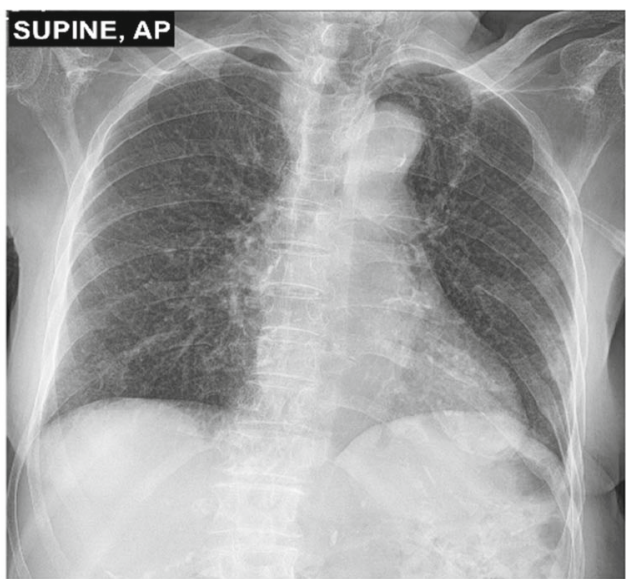

(b) Aspiration Pneumonia

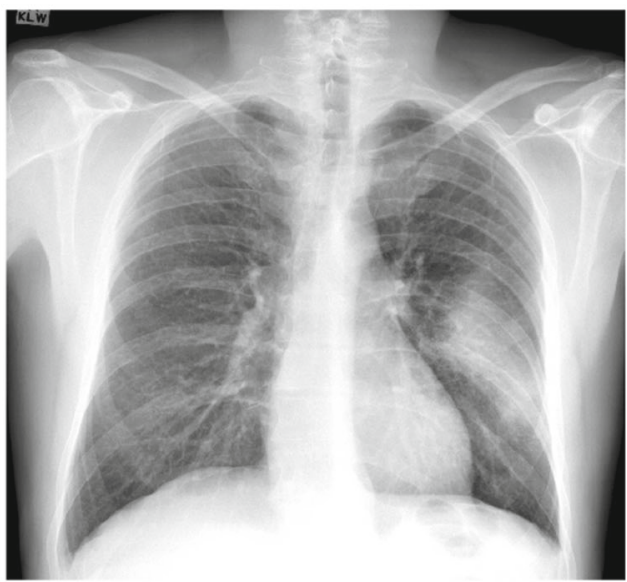

(d) Chlamydia Pneumonia

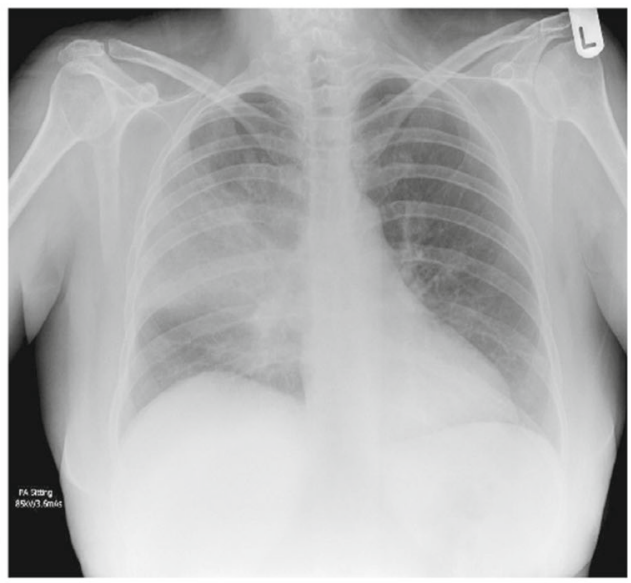

(f) Pneumococcal Pneumonia
The Renyi Fractal Dimensions or GFD of order $q \in$ $(-\infty, \infty)$ such that $q \neq 1$ of the experimental image of the probability distribution constructed as

$$
D_{q}=\lim _{\varepsilon \rightarrow 0} \frac{1}{q-1} \frac{\log _{2}\left(\sum_{i=1}^{N} p_{i}^{q}\right)}{\log _{2} \varepsilon}
$$

Here $D_{q}$ is called the generalized Renyi Entropy.

\subsubsection{Particular cases of GFD}

(a) When $q=0$, then

$$
D_{0}=-\frac{\log _{2} N}{\log _{2} \varepsilon}
$$




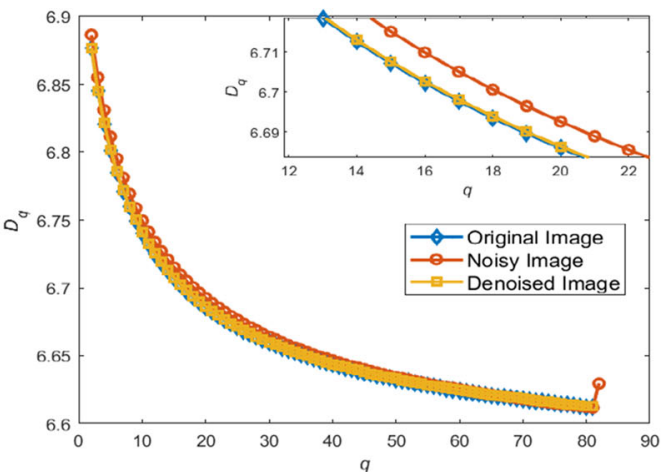

(a) Normal

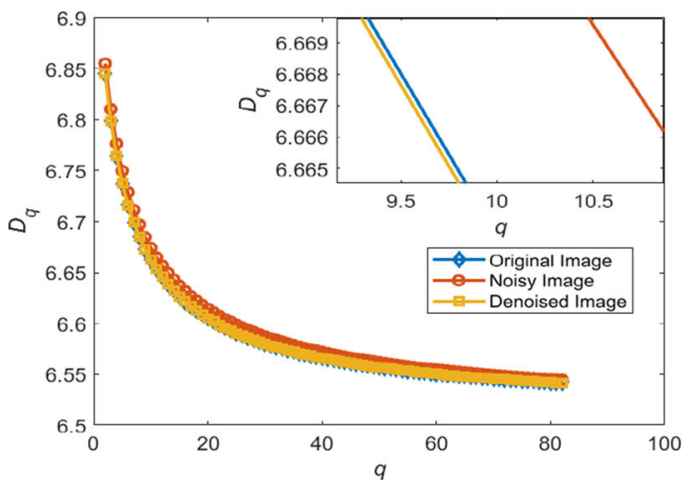

(c) Pneumocystis Pneumonia

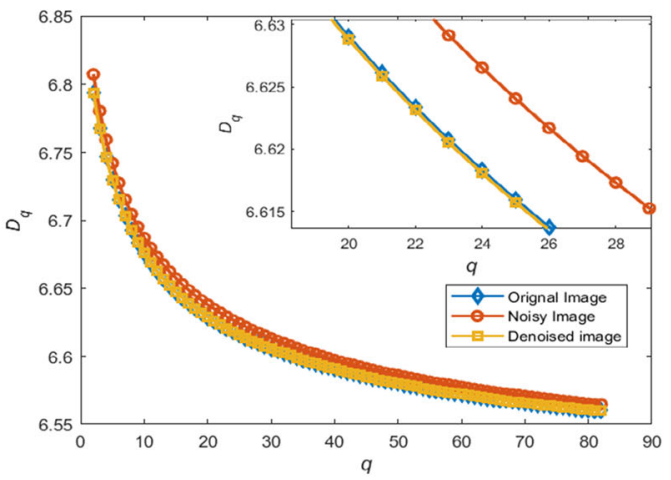

(e) ARDS

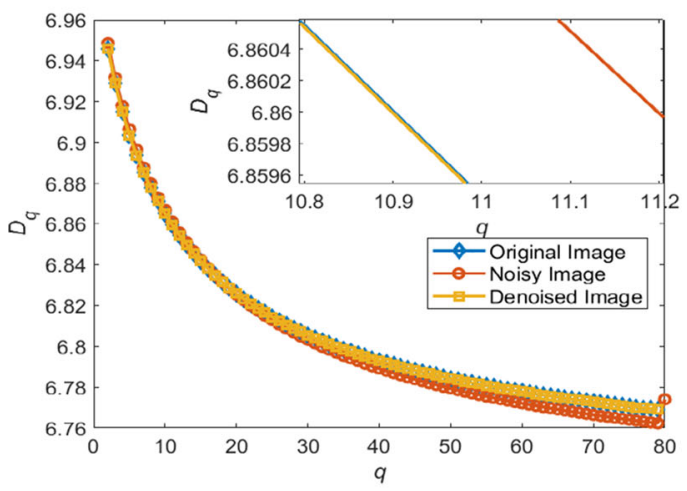

(g) Cavitating Pneumonia

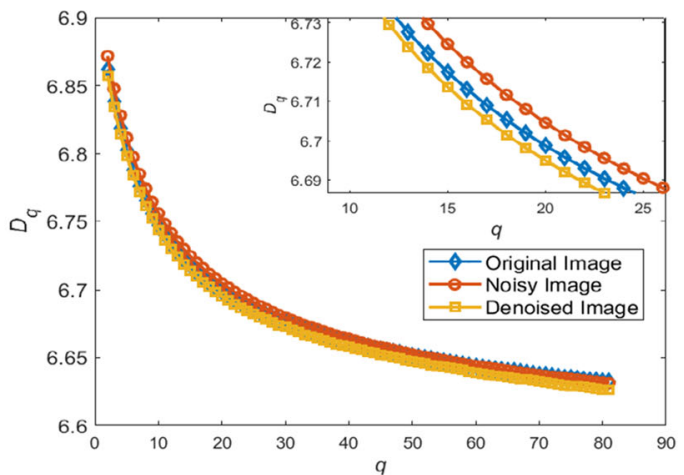

(b) COVID-19

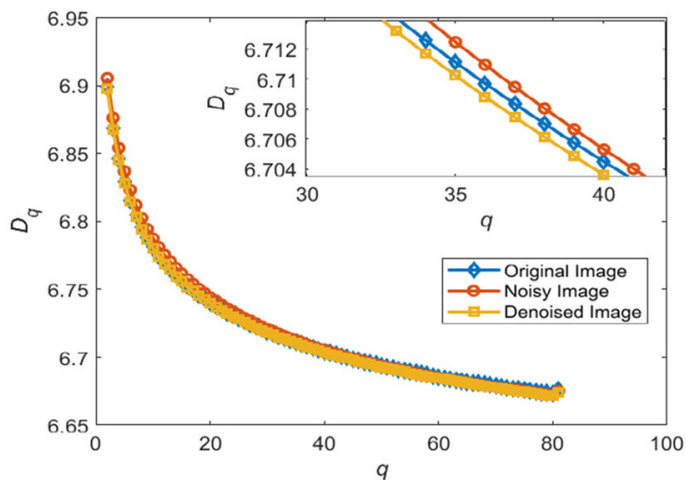

(d) Aspiration Pneumonia

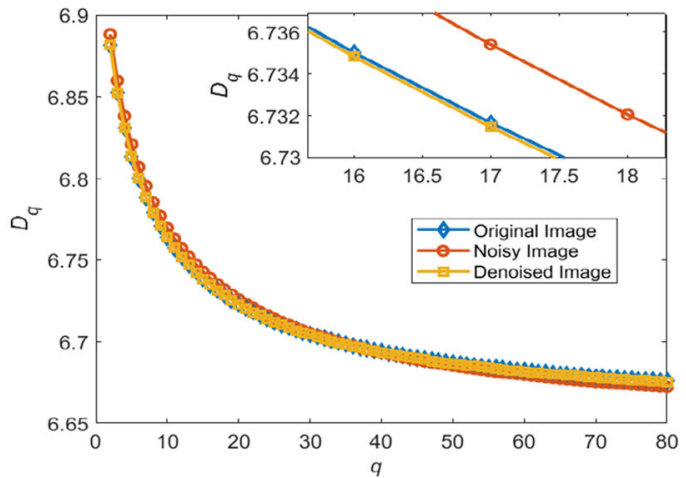

(f) Chlamydia Pneumonia

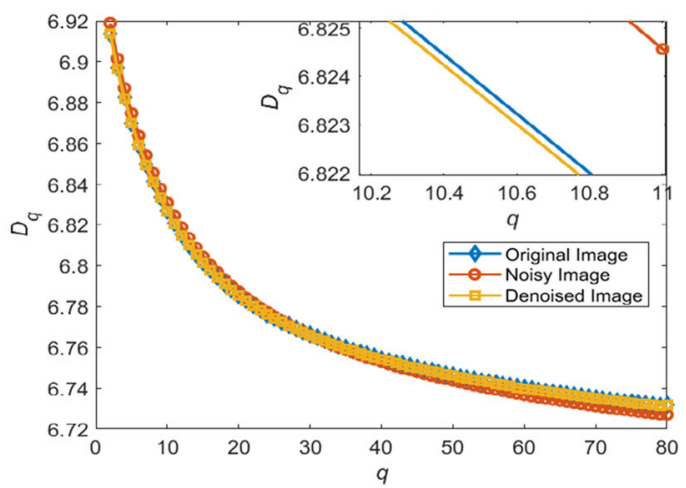

(h) Pneumococcal Pneumonia

Fig. 3 Generalized fractal dimensions spectra for original, noisy and denoised X-Ray images 
Then this is called the Fractal Dimension of the image.

(b) If $q \rightarrow 1, D_{q}$ converges to $D_{1}$, which is given by

$$
D_{1}=\lim _{\varepsilon \rightarrow 0} \frac{\sum_{i=1}^{N} p_{i} \log _{2} p_{i}}{\log _{2} \varepsilon},
$$

which is the Information Dimension of the image.

(c) If $q=2$, then $D_{q}$ is known as Correlation Dimension of the image.

(d) In this particular, there exist two limit cases of the image when $q=-\infty$ and $q=\infty$, which is defined as

$$
\begin{aligned}
D_{-\infty} & =\lim _{\varepsilon \rightarrow 0} \frac{\log _{2}\left(p_{\min }\right)}{\log _{2} \varepsilon} \\
D_{\infty} & =\lim _{\varepsilon \rightarrow 0} \frac{\log _{2}\left(p_{\max }\right)}{\log _{2} \varepsilon}
\end{aligned}
$$

where

$$
\begin{aligned}
p_{\min } & =\min \left\{p_{1}, p_{2}, \ldots, p_{N}\right\} \\
p_{\max } & =\max \left\{p_{1}, p_{2}, \ldots, p_{N}\right\}
\end{aligned}
$$

\subsection{Median filter}

Image noise is random variation of brightness or color information in images, and is usually an aspect of electronic noise. It can be produced by the sensor of a scanner or digital camera [26]. Median filtering is a nonlinear activity that is regularly used to enhance the image quality by reducing the salt and pepper noise. Such noise reduction is a preparatory step to improve the accuracy in the post-processing analysis [28].

\subsection{Qualitative measures}

The Mean Absolute Error (MAE) and the Peak Signalto-Noise Ratio (PSNR) are used to evaluate the performance of the denoising process through the median filter in gray scale images. These quality indicators [26] are defined as:

$$
\begin{aligned}
\mathrm{MAE} & =\frac{\sum_{j=1}^{N} \sum_{i=1}^{M}\left|I_{O}(i, j)-I_{F}(i, j)\right|}{M \cdot N} \\
& \text { and } \\
\mathrm{PSNR} & =20 \cdot \log \left(\frac{255}{\sqrt{\frac{1}{M \cdot N} \sum_{j=1}^{N} \sum_{i=1}^{M}\left(I_{O}(i, j)-I_{F}(i, j)\right)^{2}}}\right)
\end{aligned}
$$

where $M$ and $N$ are the dimensions of the image, $I_{O}(i, j)$ and $I_{F}(i, j)$ indicate the original image and restored image at pixel position $(i, j)$, respectively.

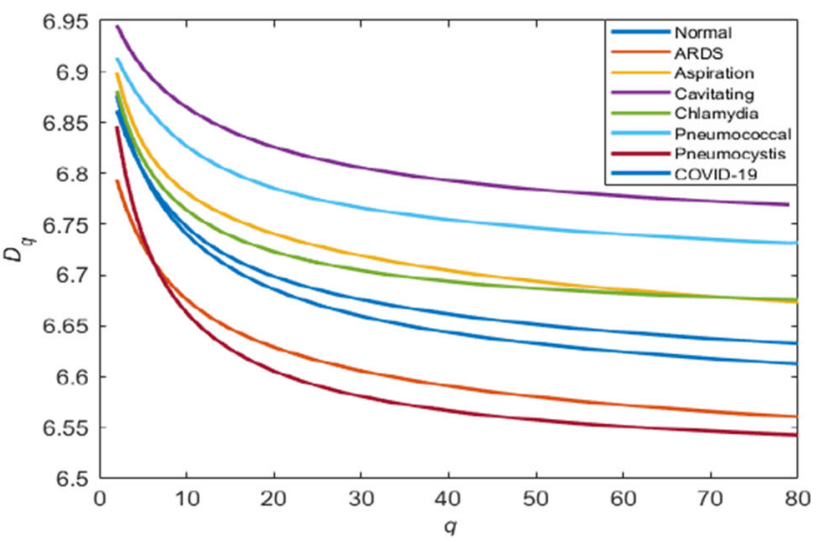

(a) Original Images

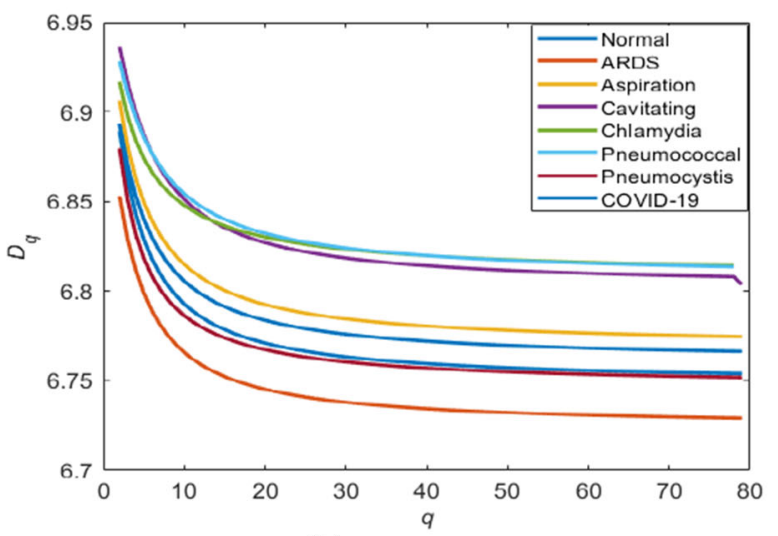

(b) Noisy Images

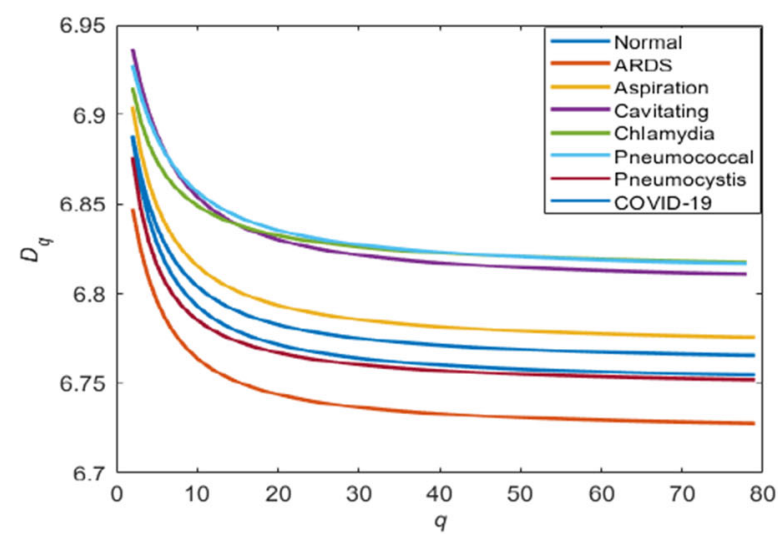

(c) Denoised Images

Fig. 4 Comparison of generalized fractal dimensions spectra for normal, COVID-19 and various pneumonia diseases

\section{Experimental data description}

In this research work, the eight grayscale X-Ray images such as Normal, COVID-19, Pneumocystis Pneumonia, Aspiration Pneumonia, Acute Respiratory Distress Syndrome (ARDS), Chlamydia Pneumonia, Cavitating Pneumonia and Pneumococcal Pneumonia are considered as tested images [38-40]. The persons/patients involved in the database of Normal, 
Table 1 Comparison of denoising performances of tested X-Ray gray scale images

\begin{tabular}{lllll}
\hline Image & $\begin{array}{l}\text { Noisy image corrupted by } \\
\text { salt and pepper noise with } \\
\text { density } 0.05\end{array}$ & PSNR & \multicolumn{2}{l}{$\begin{array}{l}\text { Denoised image using median } \\
\text { filter }\end{array}$} \\
\cline { 2 - 5 } & MAE & 18.2395 & MAE & PSNR \\
\hline Normal & 6.3670 & 18.0996 & 2.9698 & 34.5992 \\
Pneumococcal pneumonia & 6.3190 & 18.0669 & 0.9735 & 35.1402 \\
Pneumocystis pneumonia & 6.3674 & 17.7721 & 2.1847 & 37.1039 \\
Chlamydia pneumonia & 6.3741 & 17.9077 & 0.9145 & 37.2445 \\
ARDS & 6.3760 & 18.3717 & 1.0102 & 39.0648 \\
Cavitating pneumonia & 6.3967 & 18.2063 & 4.3590 & 42.6277 \\
Aspiration pneumonia & 6.4077 & 18.0406 & 2.1558 & 30.5229 \\
COVID-19 & 6.3799 & & 25.0517 \\
\hline
\end{tabular}

COVID-19, ARDS, Pneumocystis Pneumonia, Chlamydia Pneumonia, Cavitating Pneumonia are males with the age above 40; the patient of Pneumococcal Pneumonia is female at the age of 40 and the Aspiration Pneumonia patient is female at the age of 80 . A sample of X-Ray images from the healthy person and COVID19 patient are plotted in Fig. 1 and the X-Ray images for other pneumonia diseases are depicted in Fig. 2.

\section{Results and discussion}

The simulations are carried out in MATLAB software by using representative X-Ray grayscale images and demonstrated the same in this section to highlight the complexity of noise level in images for normal, COVID19 and other pneumonia types of diseases. Figures 1 and 2 are considered as sample X-Ray images for a normal healthy person and also for all the diseases like COVID-19 and other pneumonia types of diseases such as Pneumocystis Pneumonia, Aspiration Pneumonia, ARDS, Chlamydia Pneumonia, Cavitating Pneumonia and Pneumococcal Pneumonia. The distributed white areas within the lungs in figures corresponds to the infected zones. Casually, we cannot observe any major differences from these eight types of images. The white areas in these X-Ray images indicate the extreme impact of the disease.

Initially, the representative grayscale images are debased by the salt and pepper noise with density 0.05 . Afterwards, the debased images are denoised by the filtration method through the median filter. The generalized fractal dimensions for original, noisy and denoised categories of all 8 representative X-Ray images are calculated and projected in Fig. 3. In Fig. 3, it is concluded that the GFD curve for denoised image is coincided with the original image almost for all categories of X-Rays, whereas the curve of GFD for noisy case is totally differed from the original and denoised cases in all types of images.

Further, the curves of GFD measure is sketched in Fig. 4 separately for original, noisy and denoised images to compare GFD values for all X-Rays geometrically. In Fig. 4 (a), the generalized fractal dimensions spectra for original case of all tested images are compared graphically In Fig. 4b, it is observed that the comparison between all noisy images are clearly examined. Fig. 4c evidently determines the comparison of all denoised images graphically. Therefore, Fig. 4 shows the apparent discrimination among the GFD curves of all representative X-Ray images.

Hence, Figs. 3 and 4 are the evidence that the multifractal analysis plays an efficient role in the discrimination of noisy and noise-free X-Ray images using the Generalized Fractal Dimensions.

Besides that, the MAE values for pneumocystis pneumonia, pneumococcal pneumonia and chlamydia pneumonia are very low and the same measure for cavitating pneumonia and aspiration pneumonia and are very high. Similarly, the PSNR value for pneumocystis pneumonia and pneumococcal pneumonia PSNR are approximately equal and depicts that both Pneumonia having the same level of illness. However, the PSNR value for both Cavitating Pneumonia and Aspiration Pneumonia are higher than their noisy images at 0.05 density level of noise.

The comparison of the performance level of the denoising process for all tested images is analyzed and depicted in Table 1, using the qualitative measures such as MAE and PSNR. The quality measures in Table 1 also show that the denoising process is significantly effective for all categories of tested X-Ray grayscale images. It is noted that, the median filtering technique is performed noticeably to denoise the salt and pepper noise in all X-Ray grayscale as mentioned in Table 1. It is also observed that, the MAE value of ARDS and Pneumococcal Pneumonia images indicates the effectiveness of the denoising level, and also represents the denoising level is not effective in Aspiration Pneumonia images. Also, the PSNR value of Cavitating Pneumonia, ARDS, Chlamydia Pneumonia, and Pneumocystis Pneumonia are relatively higher than the other images. Mainly, the PSNR value for COVID-19 X-Ray image is lower than the other representative images and also it is implied that the denoising process in COVID-19 
images is not much effective due to non-uniformity in the affected zones.

\section{Conclusion}

In this paper, the Generalized Fractal Dimensions is obtained for all X-Ray grayscale images for the Corona Virus and the other pneumonia diseases to analyze the complexity of COVID-19 dynamics. The multifractal measure, GFD is calculated for the original, noisy and denoised images of COVID-19 and other diseases. In addition, a graphical comparison of COVID-19 X-Ray image with the images of healthy and other pneumonia diseases is done to determine the level of difficulty of the illnesses in terms of GFD curvatures. It is observed that, the fatal virus disease has been hazardous than the other diseases because the quality of denoised XRay image for COVID-19 is very low with a less PSNR value, when compared with other images. This evident shows that the content of COVID-19 infection is generally vigorous than the other pneumonia diseases.

Acknowledgements The research work has been partially supported by Hunan Provincial Natural Science Foundation of China (No.: 2019JJ60017).

Data availability statement This manuscript has associated data in a data repository. [Authors' comment:[38-40].]

\section{References}

1. B.B. Mandelbrot, The Fractal Geometry of Nature (W.H. Freeman and Company, New York, 1983)

2. M.F. Barnsley, Fractals Everywhere (Academic Press, London, 2014)

3. M.F. Barnsley, SuperFractals (Cambridge University Press, New York, 2006)

4. K. Falconer, Fractal Geometry: Mathematical Foundations and Applications (John Wiley \& Sons Ltd., England, 2003)

5. G. Edgar, Measure, Topology, and Fractal Geometry (Springer, New York, 2008)

6. S. Paul, Addison, Fractals and Choas (IOP Publishing, London, 1997)

7. P. Grassberger, Phys. Lett. A 97A(6), 227-230 (1983)

8. H.G.E. Hentschel, I. Procaccia, Physica 8D(3), 435-444 (1983)

9. D. Easwaramoorthy, R. Uthayakumar, in Proceedings of the IEEE Students' Technology Symposium, IEEE Xplore, USA (2010). p. 6-11

10. D. Easwaramoorthy, R. Uthayakumar, in Proceedings of the International Conference on Computing, Communication and Networking Technologies, IEEE Xplore, USA (2010). p. 1-6

11. D. Easwaramoorthy, R. Uthayakumar, in Proceedings of the IEEE International Conference on Communication Control and Computing Technologies, IEEE Xplore, USA (2010). p. 544-549
12. D. Easwaramoorthy, R. Uthayakumar, J. Comput. Sci. 2(1), 31-38 (2011)

13. R. Uthayakumar, D. Easwaramoorthy, Fluct. Noise Lett. 11(4), 1250034 (2012)

14. R. Uthayakumar, D. Easwaramoorthy, in Proceedings of the International Conference on Computing, Communication and Networking Technologies, IEEE Xplore, USA (2012). p. $1-5$

15. R. Uthayakumar, D. Easwaramoorthy, in Proceedings of the International Conference on Emerging Trends in Science, Engineering and Technology, IEEE Xplore, USA (2012). p. 228-234

16. R. Uthayakumar, D. Easwaramoorthy, Fractals 21(2), 1350011 (2013)

17. R. Uthayakumar, D. Easwaramoorthy, Chaos, Complexity and Leadership 2012 (Springer Proceedings in Complexity, Berlin, 2012), pp. 411-422

18. P.S. Eliahim Jeevaraj, P. Shanmugavadivu, D. Easwaramoorthy, Advances in Algebra and Analysis, Trends in Mathematics (Springer, Berlin, 2018), pp. 205-213

19. D. Easwaramoorthy, P.S. Eliahim Jeevaraj, A. Gowrisankar, A. Manimaran, S. Nandhini, Int. J. Eng. Technol. (UAE) 7(4.10), 900-903 (2018)

20. S. Nandhini, D. Easwaramoorthy, R. Abinands, Int. J. Emerg. Trends Eng. Res. 8(7), 3766-3776 (2020)

21. Santo Banerjee, M.K. Hassan, Sayan Mukherjee, A. Gowrisankar, Fractal Patterns in Nonlinear Dynamics and Applications (CRC Press, Boca Raton, 2020)

22. S. Banerjee, D. Easwaramoorthy, A. Gowrisankar, Fractal functions, dimensions and signal analysis, in Understanding Complex Systems, Springer: Complexity. (Springer, Cham, 2021)

23. D. Easwaramoorthy, A. Gowrisankar, A. Manimaran, S. Nandhini, Lamberto Rondoni, Santo Banerjee, Nonlinear Dyn. 106(2), 1375-1395 (2021)

24. M. Sharma, R.B. Pachori, U. Rajendra Acharya, Pattern Recogn. Lett. 94, 172-179 (2017)

25. M. Sharma, R.B. Pachori, J. Mech. Med. Biol. 17(7), $1740003(2017)$

26. R.C. Gonzalez, R.E. Woods, S.L. Eddins, Digital Image Processing Using MATLAB (Gatesmark Publishing, Knoxville, 2020)

27. J. Chauveau, D. Rousseau, P. Richard, F. ChapeauBlondeau, Chaos Solitons Fractals 43, 57-67 (2010)

28. T. Chen, M. Kai-Kuang, L.H.C. Chen, IEEE Trans. Image Process. 8(12), 1834-1838 (1999)

29. Q. Ding, P. Lu, Y. Fan, Y. Xia, M. Liu, J. Med. Virol. 92(9), 1549-1555 (2019)

30. E. Mahase, NEWS (BMJ Publishing Group Limited, London, 2020)

31. M. Kojicic, E. Festic, O. Gajic, Bosn. J. Basic Med. Sci. $\mathbf{9}(1)$, S59-S68 (2009)

32. D.H. Dockrell, M. Moira, K.B. Whyte, T.J. Mitchell, Chest 142(2), 482-491 (2012)

33. Y.G. Son, J. Shin, H.G. Ryu, J. Dent. Anesth. Pain Med. 17(1), 1-12 (2017)

34. A.L. Laurila, L. Von Hertzen, P. Saikku, Scand. J. Infect. Dis. Suppl. 104, 34-36 (1997)

35. C.L. Kiew, A. Brahmananda, K.T. Islam, H.N. Lee, S.A. Venier, A. Saraar, H. Namazi, Fractals 28(1), 2050019 (2020) 
36. N. Tanabe, S. Muro, S. Sato, T. Oguma, A. Sato, T. Hirai, BMC Pulm. Med. 18, 144 (2018)

37. H. Namazi, Fractals 28(07), 2050129 (2020)

38. Kaggle - Chest X-Ray Images (Pneumonia) (2021) https://www.kaggle.com/paultimothymooney/ chest-xray-pneumonia
39. SIRM - Societa Italiana di Radiologia Medica e Interventistica (2021) https://www.sirm.org/2020/03/ 07/covid-19-caso-12/

40. Radiopaedia (2021) https://radiopaedia.org/ 